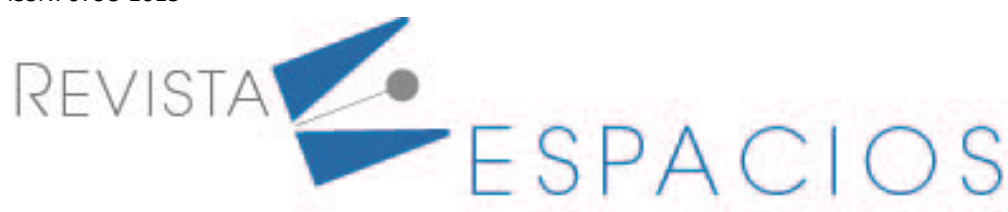

Vol. 41 (47) $2020 \bullet$ Art. 5

\title{
Diseño de un sistema acuapónico monitoreado mediante internet de las cosas e inteligencia artificial
}

\section{Design of an aquaponic system monitored by internet of things and artificial intelligence}

\author{
OVIEDO-LOPERA, Juan C. ${ }^{1}$ \\ OVIEDO-CARRASCAL, Ana I. ${ }^{2}$ \\ CARMONA-RODRIGUEZ, Claudia S. ${ }^{3}$ \\ VELEZ-SALDARRIAGA, Gloria L. ${ }^{4}$ \\ REINA-ALZATE, Jackson ${ }^{5}$
}

\begin{abstract}
Resumen
Este trabajo presenta el diseño de un sistema acuapónico, monitoreado por medio de una arquitectura de internet de las cosas e inteligencia artificial. Para la metodología se parte de un diseño acuapónico y se propone arquitectura basada en 4 capas: senado, comunicaciones, servicios y aplicaciones. Los resultados permiten la toma de decisiones con el monitoreo de variables relacionadas con aire, suelo, agua, plantas y animales. Finalmente, se espera que este diseño pueda ser implementado a diferentes escalas..
\end{abstract}

Palabras clave: acuaponía, seguridad alimentaria, internet de las cosas, inteligencia artificial

\begin{abstract}
This work presents the design of an aquaponic system, monitored by means of an internet of things architecture and artificial intelligence. The methodology is based on an aquaponic design and an architecture based on 4 layers is proposed: senate, communications, services and applications. The results allow decision making with the monitoring of variables related to air, soil, water, plants and animals. Finally, it is expected that this design can be implemented at different scales..
\end{abstract}

Key words: aquaponics, food security, internet of things, artificial intelligence.

\section{Introducción}

Colombia es un país que se ha destacado por sus actividades agropecuarias, de manera especial la producción de café, frutas y carne, especialmente la bovina; sin embargo, es un país con problemas de alimentación. Según la Encuesta Nacional de Situación Nutricional (ENSIN) realizada en el año 2015 (Minsalud, 2015) Colombia presenta problemas tanto de desnutrición como de sobrepeso. Dentro de las razones que se presentan como generadoras de dicho problema se destacan la inequidad en la producción y el acceso a los alimentos y al agua,

\footnotetext{
${ }^{1}$ Docente Investigador. Facultad de Ingeniería Agroindustrial . Universidad Pontificia Bolivariana. Colombia. Email: juan.oviedo@upb.edu.co

${ }^{2}$ Docente Investigador. Facultad de Ingeniería TICs. Universidad Pontificia Bolivariana. Colombia. Email: ana.oviedo@upb.edu.co

${ }^{3}$ Docente Investigador. Facultad de Ingeniería Agroindustrial . Universidad Pontificia Bolivariana. Colombia. Email: claudia.carmona@upb.edu.co

${ }^{4}$ Docente Investigador. Facultad de Ingeniería Agroindustrial. Universidad Pontificia Bolivariana. Colombia. Email: gloria.velez@upb.edu.co

${ }^{5}$ Director de Facultad. Facultad de Ingeniería TICs . Universidad Pontificia Bolivariana. Colombia. Email: jackson.reina@upb.edu.co
} 
además de prácticas culturales incorrectas en las dietas, sobre todo en el balance de las biocomponentes básicos (carbohidratos, lípidos, proteínas, vitaminas y minerales). Dentro de esos biocomponentes básicos, las proteínas son el más costoso y de difícil acceso.

Una de las principales fuentes de acceso a la proteína es el pescado. El pescado tiene en promedio un contenido proteico aproximado entre el 15 y el $18 \%$, un valor biológico (VB) de 76 y una digestibilidad del 94\% (Fennema, 2010); Primo, 1998). Una de las alternativas para la producción de pescado es la acuaponía, tema central de este estudio. La acuaponía se define como la integración de la producción de plantas y peces en un mismo sistema, donde el agua del proceso de la piscicultura se lleva mecánicamente al cultivo hidropónico, la cual es absorbida por las plantas; dicha agua contiene material orgánico procedente de las heces y otros compuestos del metabolismo de la actividad piscícola, que son utilizados para el crecimiento de las mismas. Finalmente, el agua del cultivo de las plantas retorna nuevamente al proceso de producción de peces en una continua recirculación de agua, empleada en dos procesos que tradicionalmente se han realizado de manera independiente. Los sistemas acuapónicos, tienen como ventajas la producción de alimento animal y vegetal dentro de un mismo sistema, un uso más efectivo del agua debido a que los sistemas acuapónicos utilizan las aguas residuales de la piscicultura que es rica en desechos de peces como nutrientes para el crecimiento de las plantas (Forchino et al., 2017); adicional a lo anterior, en comparación con la agricultura tradicional, la acuaponía recircula el agua dentro del sistema que reduce la evaporación e infiltración de agua. La ONU señala que la acuaponía utiliza una séptima parte del agua, que se emplea en la agricultura tradicional (Addy et al., 2017; Goddek y Keesman, 2018). De acuerdo a lo anterior, en primera instancia, se definirá la acuaponía y se realizará la clasificación de algunos sistemas más empleados. De otro lado, se destacan que en los sistemas acuapónicos no son necesarios, en muchos casos, el empleo de fertilizantes o pesticidas químicos, adicionalmente, se pueden emplear terrenos que no sean aptos para la producción agropecuaria o se pueden implementar en las ciudades. Los sistemas acuapónicos son de fácil construcción, los cuales pueden ser implementados por familias para auto sostenimiento y/o producción, además de tener bajos costos de mantenimiento (Buzby et al., 2016; Somervillle et al., 2014). Sin embargo, este tipo de sistemas requiere vigilar y controlar constantemente los diferentes actores. El esquema general de un sistema acuapónico se muestra en la figura 1, el cual además identifica y explica la función de cada uno de sus componentes.

Para aportar en el análisis bibliográfico de los sistemas acuapónicos en el mundo, se presenta a continuación un análisis de los artículos publicados en la base de datos de revistas científicas Scopus. Para la revisión bibliográfica se realizó una consulta del término "aquaponic" en Scopus desde el año 2015 hasta junio de 2020, recuperando 342 artículos científicos que son analizados por medio de inteligencia artificial. Con el objetivo de encontrar los temas subyacentes en los documentos, los títulos de los 342 artículos son analizados por medio de técnicas de procesamiento de lenguaje natural para posteriormente ser estudiados por medio de un ejercicio de clustering (aprendizaje no supervisado de máquinas). Como resultado, se descubrieron 4 temas en los artículos, los cuales son presentados en el cuadro 1 mediante nubes de palabras (el tamaño de la letra representa la frecuencia de aparición de las palabras en los títulos. Los temas encontrados son: 1) sistemas acuapónicos con énfasis en producción, economía y sostenibilidad, resaltando los peces y el agua; 2) sistemas hidropónicos que se enfocan en la calidad del agua, la recirculación y el crecimiento de lechuga (Lactuca sativa); 3) sistemas acuapónicos donde se evalúa la transformación del nitrógeno; y 4) sistemas acuapónicos monitoreados por internet de las cosas (IOT) e inteligencia artificial (IA). 
Figura 1

Diagrama de un sistema acuapónico
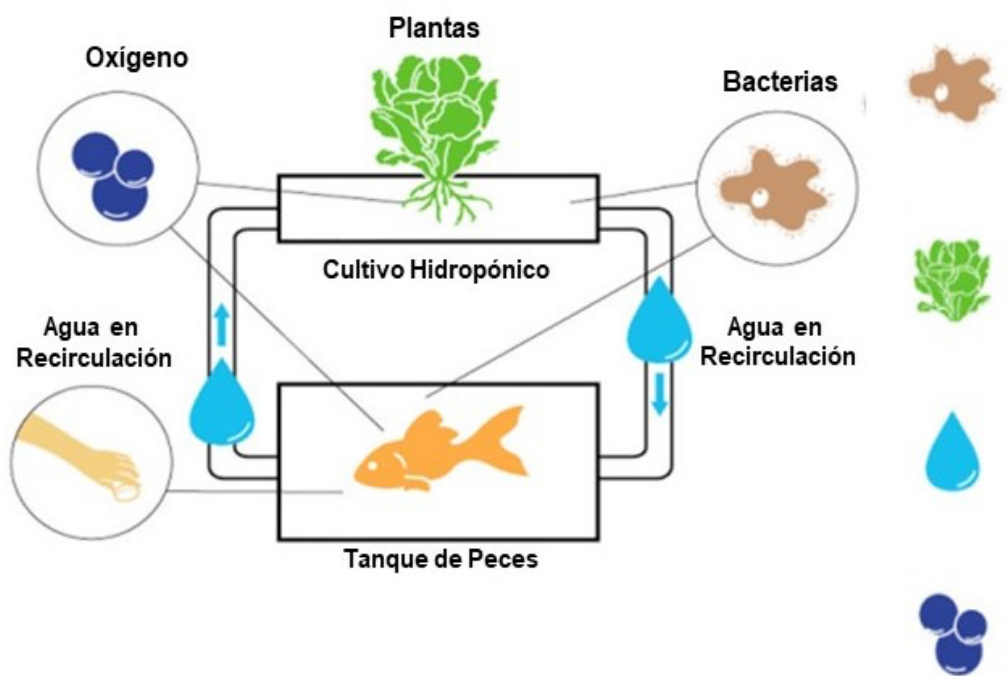

Peces: Los peces son alimentados y producen un residuo rico en amonio. Una alta cantidad de amonio en el agua es toxica para los peces, pero pueden soportar altos niveles de nitratos.

Bacterias: Las bacterias están tanto en el cultivo hidropónico como en el tanque de los peces; estas son las encargadas de transformar los nitritos en nitratos.

Plantas: Las plantas convierten los nitritos en nutrientes, los nutrientes son los fertilizantes de las plantas. Además, las raices de las plantas sirven como filtros del agua para los peces.

Agua: El agua en el sistema es filtrada a través de las plantas. El agua tiene también todos los nutrientes para los peces.

Oxígeno: El oxígeno entra al sistema a través de la bomba de aire y durante los periodos secos. Este oxigenen es esencial para el crecimiento de la planta y para la supervivencia de los peces.

Fuente: Adaptada de (Project FEED 1010, 2016)

Cuadro 1

Resultado del estudio de temas con 342 artículos de Scopus relacionados con el término "aquaponic"

\begin{tabular}{|c|c|}
\hline Nube de palabras de cada clúster & Descripción del Tema \\
\hline 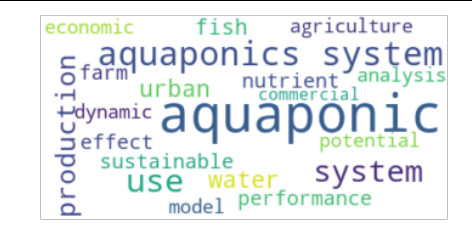 & $\begin{array}{l}\text { Investigaciones científicas cuyo tema principal es sistemas } \\
\text { acuapónicos con énfasis en producción, economía y } \\
\text { sostenibilidad, resaltando los peces y el agua. } \\
\text { Cantidad de artículos: } 125\end{array}$ \\
\hline 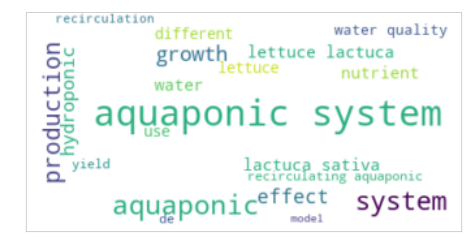 & $\begin{array}{l}\text { Investigaciones científicas cuyo tema principal es sistemas } \\
\text { hidropónicos que se enfocan en la calidad del agua, la } \\
\text { recirculación y el crecimiento de lechuga (Lactuca sativa). } \\
\text { Cantidad de artículos: } 151\end{array}$ \\
\hline 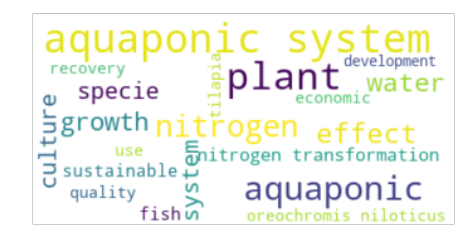 & $\begin{array}{l}\text { Investigaciones científicas cuyo tema principal es sistemas } \\
\text { acuapónicos donde se evalúa el ciclo del nitrógeno. } \\
\text { Cantidad de artículos: } 49\end{array}$ \\
\hline 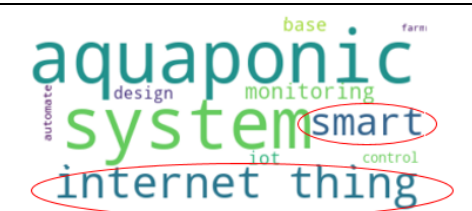 & $\begin{array}{l}\text { Investigaciones científicas cuyo tema principal es sistemas } \\
\text { acuapónicos con inteligencio y/o loT } \\
\text { Cantidad de artículos: } 17\end{array}$ \\
\hline
\end{tabular}

Fuente: Elaboración propia 
Para abordar este trabajo, se presenta a continuación los conceptos relacionados con sistemas acuapónicos, arquitecturas de IoT e IA.

\subsection{Sistemas de acuaponía}

La literatura reporta muchas configuraciones de sistemas acuapónicos; sin embargo, de forma general los sistemas acuapónicos se pueden clasificar de tres tipos: 1) Sistema de cultivo en aguas profundas, 2) Sistema de cultivo de nutrientes en películas, y 3) Sistema de cultivo de inundado y drenado. El sistema de cultivo en aguas profundas (balsa flotante) es el más empleado desde el punto de vista comercial. Las plantas se cultivan en láminas de espuma de poliestireno (o algún material liviano), que flotan en la superficie de los semilleros llenos de agua. El agua de la pecera se bombea continuamente en los semilleros y se desborda continuamente en la pecera. Los semilleros deben airearse en todo momento para evitar que se pudran las raíces. Además, el agua debe pasar a través de un filtro mecánico y biológico por separado antes de llegar a los semilleros, para eliminar cualquier partícula que pueda obstruir las raíces de la planta (Consulting Hambrey, 2013; Villadomat, 2012).

En el sistema de cultivo de nutrientes en películas, el agua rica en nutrientes se bombea hacia pequeños poros o tuberías de PCV, por las cuales fluye continuamente una pequeña cantidad de agua. El agua se bombea continuamente desde el tanque de peces a un filtro mecánico y biológico separado (para eliminar cualquier material particulado que pudiera obstruir las raíces de la planta), y luego a los tubos de crecimiento, que continuamente se drenan hacia el tanque de peces (Consulting Hambrey, 2013; Villadomat, 2012).

Finalmente, en el sistema de cultivo de inundado y drenado, las plantas se cultivan en un semillero relleno medio. El medio de soporte sirve para dos propósitos: 1) como filtro biológico y mecánico, y 2) como soporte para las plantas que pueden enraizar en él tanto como en el suelo. La cama de cultivo se llena con agua de la pecera y luego se drena. Durante la inundación, el agua y los nutrientes se introducen en la zona de la raíz de la planta. Durante el drenaje, el aire se introduce en la zona de la raíz de la planta y el agua regresa al tanque de peces (Consulting Hambrey, 2013; Villadomat, 2012).

\subsection{Internet de las cosas (IOT) para agricultura}

La agricultura de precisión busca optimizar y mejorar los procesos agrícolas para garantizar la máxima productividad y requiere mediciones distribuidas rápidas y confiables para dar a los productores una descripción más detallada de la situación actual en su área de cultivo, administrar la maquinaria automatizada, hacer un manejo eficiente de recursos como energía, agua y productos químicos para el control de plagas y el crecimiento de las plantas (Talavera et al, 2017; Quiroga et al., 2017). Para tal fin en los últimos años se han implementado un gran número de propuesta en todo el mundo (Tzounis, Katsoulas, Bartzanas, y Kittas, 2017) usando internet de las cosas IOT (Internet of Things, por su siglas en ingles) como medio para la adquisición de datos y control de actuadores sobre el proceso agroindustrial. Cada aplicación tiene factores diferenciadores de acuerdo a la extensión del proceso agrícola y los factores que se quieren medir y controlar, se han encontrado características comunes que pueden presentarse como una arquitectura que involucra aspectos desde la medición de variables en el cultivo o zona de intervención, hasta la posibilidad de monitorear y controlar remotamente y adquirir datos para predecir eventos en el comportamiento del proceso como lo presentan (Talavera et al., 2017), diversos autores lo dividen en cuatro capas (Guerrero-Ibañez et al., 2017; Gutierrez Leon et al., 2019): capa sensado o de recolección, capa de comunicaciones, capa de servicio o de gestión y capa de aplicación o consulta; sobre esta propuesta se presenta en este trabajo una propuesta para un sistema acuapónico.

\subsection{Inteligencia artificial (IA)}

La Inteligencia Artificial estudia el desarrollo de sistemas que imitan la inteligencia de los humanos y de otros seres vivos. Las principales áreas de trabajo son: 1) aprendizaje de máquinas, 2) procesamiento de lenguaje natural, 3) visión artificial y 4) robótica. El aprendizaje de máquinas (ML) (Machine Learning, por su siglas en ingles) imita la capacidad de aprendizaje de los humanos y de otros seres vivos como las colonias de hormigas. Las máquinas pueden aprender a partir de datos o de la experiencia mediante diferentes tipos de aprendizaje 
como: supervisado, no supervisado, semi-supervisado, profundo y por refuerzo (Yu y He, 2019). El aprendizaje supervisado permite desarrollar análisis predictivos de eventos futuros, por ejemplo predecir clima, predecir ventas, predecir fuga de clientes, predecir enfermedades, predecir crecimiento de animales, entre otros. El aprendizaje no supervisado permite descubrir patrones en los datos mediante la búsqueda de similitudes (clustering), búsqueda de co-ocurrencias de eventos (reglas de asociación) y selección de variables relevantes (selección de factores). El aprendizaje semi-supervisado realiza tareas predictivas cuando se tiene pocos datos disponibles para modelos supervisados y por el contrario se tienen muchos datos disponibles para modelos no supervisados. El aprendizaje profundo, es utilizado por las redes neuronales para datos complejos como imágenes y texto. Finalmente, el aprendizaje por refuerzo ha sido convencionalmente usado en los videojuegos como una inferencia basada en la experiencia de tipo premio/castigo.

El procesamiento de lenguaje natural (NLP) (Natural Languaje Processing, por su siglas en ingles) imita la capacidad de comunicación por texto y por habla (Baclic et al., 2020). Se han desarrollado diversas tareas en el PLN como clasificación de texto, agrupación de texto, extracción de información, descubrimiento de entidades, modelamiento de temas, análisis de sentimientos de las publicaciones en redes sociales, entre otros.

La visión artificial imita la capacidad de tomar decisiones a partir de imágenes o videos (Yigit, Sabanci, Toktas, y Kayabasi, 2019). Algunos ejemplos son las cámaras inteligentes, los sistemas de foto-multas, reconocimiento de rostros, supervisión de cultivos inteligentes mediante cámaras, entre otros. Por último, la robótica imita el movimiento y comportamiento de los humanos y de otros seres vivos, dotando a los robots de la capacidad de tomar decisiones con inteligencia para desarrollar diferentes tareas (Gao, Wanyama, Singh,Gadhrri y Schmidt, 2020).

Por lo anterior, en este trabajo se propone el diseño de un sistema acuapónico, monitoreado por una arquitectura de loT e inteligencia artificial, que permita la adquisición de datos de las condiciones de cultivo y la visualización de la información a través de un dispositivo móvil.

\section{Metodología}

Para llevar a cabo esta propuesta de diseñar un sistema acuapónico monitoreado mediante loT e IA, se emplea un método cualitativo, no experimental y descriptivo. La propuesta se desarrolló en las siguientes fases:

\subsection{Fase 1}

Identificación de Requerimientos: en esta fase se identificaron los requerimientos relacionados con las cuatro capas del sistema que son: (1) Capa de sensado, (2) Capa de comunicaciones, (3) Capa de servicios y (4) Capa de aplicaciones. Para esta fase, se realizó una investigación sobre diferentes modelos y arquitecturas implementadas para sistemas acuapónicos o sistemas similares como son los sistemas hidropónicos, sistemas que permitieran la generación de información en tiempo real buscando: (a) optimizar el proceso, (b) disminuir el riesgo y (c) controlar diferentes variables, las cuales pueden incidir en la producción, con ello se busca apoyar los procesos de toma de decisiones de manera más acertiva.

\subsection{Fase 2}

Definición de la arquitectura: en esta fase se evaluaron las condiciones de los sistemas acuapónicos, así como las necesidades de información identificadas en la fase 1. Luego se procedió a definir la arquitectura del sistema, identificando los sensores necesarios y los sistemas de comunicación entre los dispositivos, que permitiera la captura de información en tiempo real, de manera eficiente y segura. Se incluyeron los sistemas de control de riego, de fertilización, de acceso, de plagas y de iluminación, todos ellos, elementos importantes para la gestión del sistema acuapónico. Posteriormente, con la arquitectura propuesta, se procedió a definir los servicios que se necesitaban para el sistema acuapónico.

\subsection{Fase 3}


Definición de Servicios: en esta fase se definieron los servicios necesarios para el monitoreo del aire, del suelo, del agua, de las plantas y de los animales del sistema acuapónico. En esta fase se definió como se iba a gestionar el flujo de datos enviados desde la capa de comunicación para su almacenamiento y la seguridad de la información en el proceso de captura de datos. Uno de los principales servivios a definir está relacionado con la inteligencia artificial para la toma de decisiones sobre el sistema.

\subsection{Fase 4}

Definición de aplicaciones: en esta fase se diseñaron aplicaciones para la arquitectura de loT propuesta, que permita la adquisición de datos de las condiciones de cultivo y la visualización de la información a través de un dispositivo móvil. Esta fase le brinda información adicional y genera valor agregado al sistema en sí, porque ofrece información de utilidad para los usuarios y apoya el proceso de toma de decisiones en tiempo real. También se definieron aplicaciones para el usuario final, que le permite ver el comportamiento del sistema acuapónico, el avance de las plantas y los animales y el comportamiento del entorno, facilitando al usuario la toma de decisiones.

\section{Resultados}

Con el objetivo de diseñar un sistema acuapónico monitoreado mediante loT e IA, a continuación se presenta la arquitectura del sistema y la construcción de un prototipo del servicio de inteligencia artificial.

\subsection{Arquitectura propuesta para un sistema de acuaponía monitoreado por loT e IA}

A continuación, se presenta el diseño de un sistema acuapónico basado en 4 capas: sensado, comunicaciones, servicios y aplicaciones. Estas capas facilitan la toma de decisiones en el sistema de acuaponía, con un monitoreo de variables relacionadas con el aire, suelo, agua, plantas y animales como se presenta en la figura 2.

Figura 2

Arquitectura propuesta para

el sistema de acuaponía

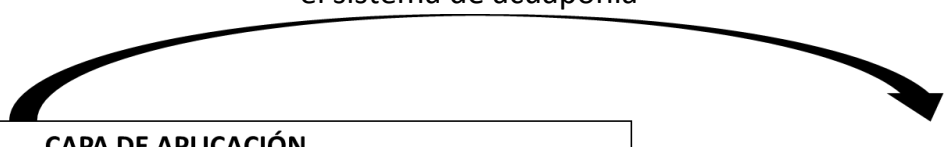

\section{CAPA DE APLICACIÓN}

- Aplicaciones que permiten la adquisición, tratamiento y gestión de la información para una correcta toma de decisiones.

\begin{tabular}{l} 
CAPA DE SERVICIOS \\
- Servicio de comunicación \\
- Servicio de almacenamiento en la nube o local \\
- Servicio de visualización \\
- Servicio de inteligencia artificial \\
\hline
\end{tabular}

\section{CAPA DE COMUNICACIONES}

- Comunicación entre nodos sensores y nodo de enlace (ZigBee, WiFi, Bluetooth)

- Comunicación entre el nodo de enlace y los datos en nube (cualquier tipo de sistema de comunicaciones para redes de área metropolitana ya sean cableadas o inalámbricas)

\section{CAPA DE SENSADO}

Tecnologías inalámbricas como redes de sensores inalámbricas (WSN), identificación por radio frecuencia (RFID) y dispositivos NFC.

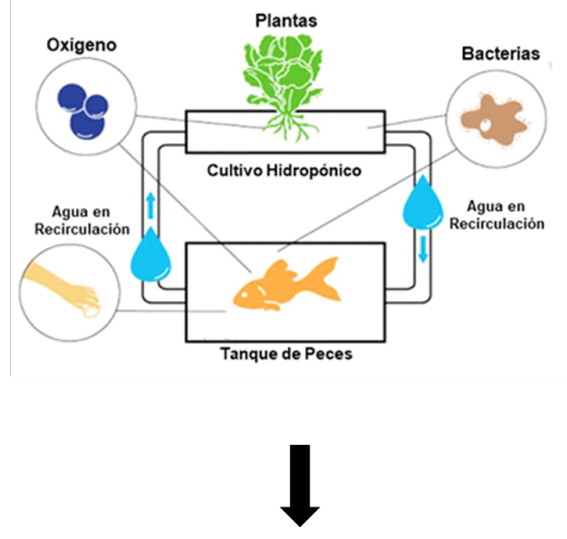

- Monitoreo del aire

- Monitoreo del suelo

- Monitoreo del agua

- Monitoreo de plantas

- Monitoreo de animales

Fuente: Elaboración propia 


\section{Capa de sensado}

En la capa de percepción o sensado se utilizan tecnologías inalámbricas como redes de sensores inalámbricas (WSN, por su siglas en ingles), identificación por radio frecuencia (RFID, por sus siglas en ingles) y dispositivos NFC. Los dispositivos ubicados en esta capa están enfocados en medir, realizar un procesamiento básico, controlar y establecer algún tipo de comunicación (Tzounis, Katsoulas, Bartzanas y Kittas, 2017; Aqeel-UrRehman, Abbasi, Isla y Shaikn, 2014). Una de las características más relevantes de este tipo de elementos es que poseen baja potencia, por lo que sus actividades deben programarse para hacer un uso adecuado de la energía (Tzounis et al., 2017). Esta es una de las principales razones por las cuales se hace referencia a que solo se hace un procesamiento básico, enfocado principalmente al acondicionamiento de las variables para su transmisión.

A continuación, se describen las áreas en las que se aplican el monitoreo de variables:

- Monitoreo del aire: proporciona mediciones periódicas o continuas, para evaluar y determinar parámetros ambientales o niveles de contaminación y tratar de evitar efectos negativos y perjudiciales (Talavera et al., 2017). En este caso las variables que se miden frecuentemente son: temperatura, humedad, presión atmosférica, velocidad del viento y dirección del viento(Aqeel-Ur-Rehman, Abbasi, Islam y Shaikh,2014).

- Monitoreo del suelo: los sistemas propuestos se utilizan para monitorear permitividad dieléctrica, nivel de lluvia, nivel de agua, conductividad, salinidad (Aqeel-Ur-Rehman et al., 2014), la temperatura, y humedad del suelo de múltiples capas en un campo de tierras de cultivo (Talavera et al., 2017).

- Monitoreo del agua: tienen la intención de monitorear la contaminación del agua o la calidad del agua mediante la detección de sustancias químicas, el pH y la temperatura, que pueden alterar el estado natural del agua(Talavera et al., 2017), para el proyecto debido a la aplicación con peces se vuelve fundamental el monitoreo de estas variables.

- Monitoreo de plantas: En este sentido se registran sensores para medir fotosíntesis, radiación solar humedad, hidrogeno, $\mathrm{CO}_{2}$ y temperatura(Aqeel-Ur-Rehman et al., 2014)(Talavera et al., 2017).

- Monitoreo de animales: Este subdominio se refiere al rastreo de animales para determinar la posición, el comportamiento y los patrones de migración (Talavera et al., 2017). En el caso de la acuaponía este tipo de variable no será sensada dado que se conoce la posición de los peces por el tipo de aplicación que se propone.

Para el sistema de control, se utilizan redes con actuadores para diferentes fines:

- Control de riego: se define como la aplicación artificial de agua en tierras agrícolas y se considera como uno de los componentes más importantes de la agricultura. La escasez de agua en varias áreas instiga la necesidad de un uso adecuado del agua que debe suministrarse solo a aquellos lugares donde se necesita y en la cantidad requerida(Aqeel-Ur-Rehman et al., 2014). La mayoría de las aplicaciones se concentran en reducir el desperdicio de agua (Talavera et al., 2017). Para sistemas acuapónicos este tipo de actuadores se ubican no solo para el riego del cultivo, sino también para permitir la recirculación de agua, la cual tiene fases intermedias de filtrado.

- Fertilización: Los fertilizantes se utilizan para aumentar la fertilidad del suelo que afecta directamente crecimiento vegetal y calidad de los alimentos (Aqeel-Ur-Rehman et al., 2014). Los trabajos recientes se enfocan en la aplicación de prácticas de conservación para mejorar el uso de nutrientes, la eficiencia, la calidad de los cultivos, el rendimiento general y el rendimiento económico al tiempo que reducen el transporte de nutrientes fuera del sitio (Talavera et al., 2017). Dentro del sistema acuapónico se utilizan los sedimentos en agua generados por los peces para nutrir orgánicamente el cultivo sin necesidad de sustancias adicionales. En la acuaponía este sistema de control va enlazado con el control de riego.

- Control de plagas: se refiere a las estrategias de manejo de plagas agrícolas El control de plagas podría ser más sostenible cuando las prácticas agrícolas sean más compatibles con los sistemas ecológicos (Aqeel-Ur-Rehman et al., 2014). Las aplicaciones de loT se han implementado dentro de invernaderos y su uso dependerá del ambiente o zona donde se ubique el sistema acuapónico. 
- Control de acceso: en este sentido se han registrado trabajos para detectar la presencia de intrusos dentro de sistema agrícola (Talavera et al., 2017) o para controlar la posición de tránsito de animales a través de las áreas de pasto (Aqeel-Ur-Rehman et al., 2014). Para dichas propuestas se requirió de sistemas de posicionamiento (GPS) y redes celulares para envío de alertas. Por las condiciones del sistema acuapónico este no es un tipo de control de interés durante esta investigación, pero no se descarta que a futuro sea necesario incluirlo.

- Control de iluminación: Se ha utilizado en invernaderos (Tzounis et al., 2017) para la horticultura y viticultura; su uso en el proyecto dependerá del ambiente o zona donde se ubique el sistema acuapónico.

Actualmente, la mayoría de las aplicaciones de monitoreo prefieren baterías recargables conectadas a paneles solares, que ofrecen un suministro de energía simple pero sostenible; sin embargo, para sistemas de control es necesario tener sistemas de potencia para alto consumo dependiendo del tipo de actuador que se desee controlar (Talavera et al., 2017).

\section{Capa de comunicaciones}

En esta capa se analizan los sistemas de comunicaciones, teniendo en cuenta: 1) la interconexión de los nodos de sensado, los actuadores y el nodo de enlace; y por otro lado, 2) la conexión entre el nodo de enlace y los sistemas de procesamiento y análisis de datos en la nube. En cada una de estas etapas se utilizan tecnologías diferentes las cuales se describen a continuación. La conectividad a Internet se convirtió en la norma para muchas aplicaciones comerciales y hoy es parte integral de muchas empresas, productos industriales y de consumo para proporcionar acceso a la información. loT se enfoca en el uso de Internet en el que las cosas físicas se comunican (Cor, Sjaak y Bedir, 2016). Una arquitectura de loT se puede subdividir en un nivel de dispositivos, nivel de red y nivel de aplicación (Atzori, Lera, y Morabito, 2010) (Verdouw et al., 2016).

El nivel de dispositivos o de control y percepción, proporciona capacidades para (1) Identificación automática (también denominada AutolD), (2) Detección y (3) Activación de actuadores.

Las tecnologías más importantes de AutolD utilizadas en la agricultura incluyen códigos de barras y RFID. La RFID se ha convertido en una práctica común en la identificación y seguimiento de los animales (Scalera et al, 2013) y también se ha utilizado en la cadena alimentaria para el control de la trazabilidad (Zhao et al, 2015) (Kubo, 2015). Además de los dispositivos AutolD, en aplicaciones agrícolas y acuapónicas, se emplean una gran cantidad de sensores diferentes que permiten medir propiedades dinámicas de elementos físicos, incluidas condiciones tales como la temperatura, humedad, iluminación, $\mathrm{CO}_{2}$, amoníaco y valores de $\mathrm{pH}$. La detección de objetos es compatible con dispositivos móviles, como lectores de código de barras / RFID y teléfonos inteligentes, que permiten a los seres humanos realizar acciones adicionales, como inspecciones de calidad visual. Los actuadores se utilizan para operar de forma remota objetos tales como control de clima, riego, refrigeración, sistemas de iluminación, sistemas de control de acuicultura y acuaponía. Sin embargo, la mayoría de los artículos revisados se centra en la detección, mientras que la actuación y el control remoto solo se abordan en algunos documentos, tales como (Ran, 2014; Tao et al, 2014; Odema, Adly, Wahba y Ragai, 2018).

En la comunicación entre nodos sensores y nodo de enlace, dada la aplicación que se desea con el proyecto en sistemas acuapónicos, se usan las tecnologías que son más utilizadas en sistemas tipo invernadero (Talavera et al., 2017), las cuales se asemejan por el área del cobertura de la red y que están dentro de un ambiente controlado, denominadas redes de área personal inalámbricas (WPAN, por su siglas en ingles)); uno de los grandes desafíos es la interferencia entre dispositivos que operan en la misma o en bandas vecinas(Tzounis et al., 2017):

- ZigBee: La tecnología define la red y los protocolos de la capa de aplicación basados en el estándar IEEE 802.15 (Institute of Electrical and Electronics Engineers, por sus siglas en ingles). Es eficiente en el consumo de energía, de bajo costo y confiable, esta tecnología es preferida para aplicaciones en los dominios agrícolas. Los dispositivos habilitados para ZigBee tienen un ciclo de trabajo bajo y, por lo tanto, son adecuados para aplicaciones agrícolas, como la gestión del riego, el control de plaguicidas y 
fertilizantes, la gestión de la calidad del agua, donde se requiere una actualización periódica de la información. Sin embargo, las aplicaciones ZigBee producen velocidades de datos bajas de solo 20-40 kbps y 250 kbps a $868 / 915 \mathrm{MHz}$ y frecuencias de $2,4 \mathrm{GHz}$ de la banda ISM, respectivamente.

- WiFi: Es una conexión inalámbrica a Internet basada en la familia de estándares IEEE 802.11 (IEEE 802.11, $802.11 \mathrm{a} / \mathrm{b} / \mathrm{g} / \mathrm{n}$ ). Actualmente, es la tecnología inalámbrica más utilizada en dispositivos que van desde teléfonos inteligentes y tabletas hasta computadoras de escritorio y portátiles. WiFi proporciona un rango de comunicación decente del orden de 20 m (interior) a $100 \mathrm{~m}$ (exterior) con una velocidad de transmisión de datos del orden de 2-54 Mbps. En aplicaciones agrícolas, WiFi amplía el uso de arquitecturas heterogéneas que conectan múltiples tipos de dispositivos a través de una red ad-hoc (Ojha et al., 2015a).

- Bluetooth: se basa en el estándar IEEE 802.15.1, es una tecnología inalámbrica de bajo consumo y bajo costo utilizada para la comunicación entre dispositivos portátiles y computadoras de escritorio en un corto rango (8-10 m). La velocidad de datos lograda en varias versiones de Bluetooth varía de 1 a 24 Mbps. Las ventajas de esta tecnología son su naturaleza ubicua, y por lo tanto, es adecuada para su uso en aplicaciones agrícolas de varios niveles. La versión de este estándar de bajo consumo de energía se denomina Bluetooth Low Energy (BLE) La topología BLE admite conexiones uno-a-uno y uno-a-muchos entre dispositivos (Ojha et al., 2015a).

En la comunicación entre el nodo de enlace y los datos en nube, para este tipo de conexión podría usarse cualquier tipo de sistema de comunicaciones para redes de área metropolitana ya sean cableadas o inalámbricas, sin embargo debido al ambiente rural y los costos que implican las conexiones cableadas, las implementaciones encontradas usan tecnologías celulares para este tipo de conexiones (Talavera et al., 2017). El estándar sistema global para las comunicaciones móviles (GSM, por sus siglas en Ingles) se divide además en la red de acceso de radio GSM EDGE (GERAN) y la red de acceso de radio terrestre UMTS (UTRAN). Numerosos dispositivos inalámbricos se han desarrollado según los diversos estándares inalámbricos. Como en muchos aspectos de loT, la interoperabilidad es el mayor desafío (Tzounis et al., 2017).

\section{Capa de servicios GSM EDGE (GERAN)}

La capa de servicio gestiona el flujo de datos enviados desde la capa de comunicación para su almacenamiento, análisis, visualización y seguridad. Dentro de esta capa se ubican los siguientes servicios:

\section{Servicio de conexión}

Según (Sundmaeker et al, 2016) (Pérez-Freire et al., 2015), cada dispositivo debe ser identificable de manera única, equipado con sensores y conectado en tiempo real a Internet, para:

- Mejor detección y monitoreo de la producción, incluido el uso de los recursos agrícolas, el desarrollo de los cultivos, el comportamiento de los animales y el procesamiento de alimentos;

- Una mejor comprensión de las condiciones agrícolas específicas, como el clima y las condiciones ambientales, la aparición de plagas, malezas y enfermedades;

- Un control más sofisticado y remoto de las operaciones de granja, procesamiento y logística por sensores, actuadores y robots, por ejemplo, en aplicación precisa de pesticidas y fertilizantes, robots para el deshierbe automático;

- Mejorar el seguimiento y la trazabilidad de la calidad de los alimentos mediante el control remoto de la ubicación y las condiciones de los envíos y productos;

- Aumentar la conciencia del consumidor sobre la sostenibilidad y los problemas de salud a través de nutrición personalizada, dispositivos vestibles y domótica.

La comunicación con el resto de la cadena de suministro se realiza a través de mensajes electrónicos EDI o XML, generalmente en un enfoque orientado a los servicios. En particular, la aplicación de Wireless Sensor Networks (WSN) en la agricultura ha recibido mucha atención como se muestra en algunos documentos de autores a los que se sugiere se consulten como (Badia-Melis et al., 2015), (Aqeel-Ur-Rehman et al., 2014) y (Ojha et al., 2015b). 


\section{Servicio de almacenamiento}

Aunque el almacenamiento en la nube representa un servicio clave para los sistemas de loT, la mayoría de los investigadores prefieren su propia implementación de almacenamiento de datos (Talavera et al., 2017). Sin embargo, en la actualidad la nube ofrece valiosos servicios, incluyendo análisis de datos que benefician este tipo de sistemas. Existen diversas aplicaciones donde la fusión de datos se realiza en la nube, un ejemplo moderno de soluciones Cloud loT para el monitoreo del clima y la optimización del clima basadas en servicios de análisis en la nube se describe en (Tzounis et al., 2017), donde los datos provienen de diversas fuentes como sensores dentro y fuera de las instalaciones, estaciones meteorológicas, datos históricos de bases de datos. El usuario puede interactuar de forma remota con el sistema a través de una amplia variedad de dispositivos (computadoras portátiles, tabletas, teléfonos inteligentes, etc.). Otro ejemplo de arquitectura para agricultura basada en la nube es FarmBeats de Microsoft (Microsoft, 2015), la cual consiste en drones UAV y sensores, soporte de conectividad e infraestructura en la nube que incluye análisis de back-end basados en el aprendizaje automático con funciones predictivas y almacenamiento en la nube. En el caso de sistemas asociados a agricultura de precisión en general y sistemas acuapónicos en particular, se recomienda disponer de servicios de almacenamiento en nube, básicamente por la carencia de infraestructura para implementar almacenamiento in situ y por el nivel de cualificación de las personas a cargo del sistema.

\section{Servicio de visualización}

De forma general, se han aplicado diferentes estrategias de visualización basadas en web, móvil y local. La visualización va enfocada en cuatro subdominios: monitoreo, control, predicción y logística (Talavera et al., 2017).

\section{Servicio de inteligencia artificial}

Una vez que los datos llegan a la capa de servicios, se pueden alimentar modelos de inteligencia artificial que faciliten la toma de decisiones mediante algoritmos de aprendizaje de máquinas y de visión artificial:

- Con aprendizaje supervisado de máquinas se puede realizar la predicción de variables ambientales e identificar las fuentes de contaminación a través del tiempo y el espacio, lo que lleva a decisiones más rápidas y mejores para garantizar un ambiente seguro y saludable para el sistema (Talavera et al., 2017). También se puede realizar la predicción de cultivos y decidir la mejor secuencia con la información actual de nutrientes del suelo (Channe et al., 2015). En este tipo de aprendizaje se requiere un histórico de datos etiquetados para realizar la inferencia (Oviedo, et al.,, 2015). Las técnicas más usuales en la aplicación de tareas predictivas son: árboles de decisión, redes neuronales, máquinas de soporte vectorial, métodos bayesianos, métodos de regresión y métodos perezosos (vecinos más cercanos) y métodos de ensamble (sistemas de votación de varios modelos).

- Con aprendizaje no supervisado de máquinas se pueden descubrir patrones ocultos que permitan describir los datos, por ejemplo encontrar tipos de suelos, tipos de peces o tipos de plantas. Las técnicas más usuales son: algoritmo K-means para análisis de clustering, algoritmo Apriori para la creación de reglas de asociación y Correlaciones para el análisis de factores.

- Con imágenes capturadas del sistema acuapónico, también se pueden realizar tareas de visión artificial como pronosticar el peso de los peces a partir de una foto o pronosticar el estado de las plantas. Para analizar las imágenes o videos se deben realizar tareas de representación mediante histogramas de color, textura o características lógicas de las imágenes donde se detecten figuras y áreas.

\section{Servicio de seguridad}

Los sistemas de loT deben garantizar la confidencialidad, integridad y disponibilidad, para cumplir con los elementos básicos de la seguridad informática y de la información. Uno de los métodos para garantizar la integridad y confidencialidad de los datos transmitidos se utilizan diferentes métodos de certificados digitales, así mismo con estos métodos se pueden utilizar diferentes algoritmos de cifrado, como curvas elípticas y los que 
se consideran de criptografía ligera. En 2017 se presentó un ataque informático mundial conocido como Mirai, el cual implicó la utilización de una Botnet de dispositivos loT con el que realizaron múltiples ataques DDoS por todo el mundo, los investigadores evidenciaron la necesidad real de implementar mecanismos que permitan asegurar la integridad, la confidencialidad, la disponibilidad y la privacidad de dispositivos de loT. (Bertino y Islam, 2017)

Investigadores de la Universidad de Bristol plantean que los ecosistemas de loT representan la nueva generación del internet y son atractivos para atacantes y piratas informáticos, los investigadores proponen replantear el tradicional modelo de tres capas y establecen una nueva arquitectura basada en cuatro (sensor, red, servicio y aplicación), este grupo plantea una visión en retrospectiva que analiza la historia de la tecnología y una revisión del modelo de seguridad, privacidad y confianza, aquí analizan a detalle los problemas de seguridad conocidos para cada una de las capas de la arquitectura propuesta. (Li, Tryfonas, y Li, 2016). Uno de los trabajos más recientes relacionados con este aspecto es el de (Garcia-Morchon et al., 2017), que describe a grandes rasgos los inconvenientes de seguridad que se presenten hoy en día con respecto al uso del loT en todas sus capas, IP, aplicación, comunicación. Así mismo, muestra los diferentes grupos que están trabajando en cada uno de los campos para poder dar una solución a los inconvenientes detectados. Dentro de los problemas mencionados están los problemas de autenticación y cifrado de la información transmitida por estos dispositivos, ya que la capacidad computacional que tienen no puede soportar algoritmos robustos de cifrado.

Son Thanh Nguyen y Chunming Rong del departamento de ingeniería eléctrica y ciencias de la computación de la Universidad Stavanger de Noruega en (S. Nguyen and C. Rong, 2007), quienes proponen un sistema de Identidad basada en criptografía para los equipos y redes ZigBee. Explican que estas redes usan algoritmos de criptografía simétrica, específicamente AES. Plantean la solución de Identidad Basada en Criptografía (IBC) porque el algoritmo AES a nivel corporativo genera la misma cantidad de llaves de cifrado como la cantidad de dispositivos pertenecientes a la red, lo que llega a ser un inconveniente si la red de dispositivos es muy grande, descartan la posibilidad de utilizar la infraestructura de llave pública (PKI), ya que con IBC no se necesita un directorio de llaves.

En la propuesta de S. Nguyen and C. Rong, plantean como llave pública del dispositivo el nombre de la función que va a cumplir, adicionalmente para evitar la duplicidad de nombre le agregan la estampa de tiempo. Así mismo plantean diferentes arquitecturas para los diferentes escenarios que consideraron probables, como el uso de un dispositivo perteneciente a uno o diferentes dominios de confianza, domótica o dispositivos loT para hogares.

En el trabajo (Contreras, y Mayol, 2016), analizan el comportamiento de las curvas elípticas específicamente el algoritmo de firma digital de curvas elípticas (ECDSA), para redes de sensores (WSN). Utilizan este algoritmo ya que se pueden alcanzar mejores niveles de seguridad que con el algoritmo de RSA. "El principal atractivo en el uso de claves de menor tamaño radica en el desarrollo de algoritmos criptográficos que consumen menor cantidad de recursos computacionales tales como ciclos de procesamiento y espacio de memoria del sistema" (Contreras y Mayol, 2016).

Sanaah Al Salami, Joonsang Baek, Khaled Salah, Ernesto Damiani en (Salami, Baek, Salah y Damiani, 2016), proponen un sistema de Identidad Basada en cifrado para los dispositivos que hacen parte de una red loT de domótica. Allí plantean un método de cifrado usando IBE y cifrado de llave pública con estado (Stateful public key encryption). Se plantean realizar una variante a la manera de cifrar usada por el método de IBE, la cual es separar la llave de cifrado y el texto a cifrar. En esta propuesta, crean un nuevo esquema llamado "Cifrado ligero para casas inteligentes (Lightweight Encryption for Smart Home (LES)", en donde utilizando Diffie-Hellman con estado (Stateful Diffie-Hellman) y HASH SHA1, logran mejorar sustancialmente el procesamiento que deben realizar los dispositivos de loT para la transmisión de información entre ellos. Además, en este trabajo realizan una evaluación de este esquema utilizando IND-CPA siendo satisfactoria. 


\section{Capa de aplicación}

Finalmente, la capa de aplicación proporciona la inteligencia para tareas de control específicas basadas en objetos virtuales. En la capa de aplicaciones, se encuentran aquellas aplicaciones que permiten la adquisición, tratamiento y gestión de la información, haciendo uso de los servicios que ofrece la capa de procesamiento, le otorgan un valor agregado a la información y ofrecen algún tipo de utilidad a los usuarios. En esta capa se deben implementar interfaces dirigidas a los usuarios de la información. En esta capa se recibe la información que se adquiere por medio de los sensores y se realiza el monitoreo de la información que se captura, para luego realizar el procesamiento de la información y generar información para la toma de decisiones. En esta capa, se realiza el control a la información que se captura, para validar la información y tomar decisiones con información confiable. Ya con la información validada se pueden realizar modelos de predicción, generando información que facilita la toma de decisiones.

Por medio de esta capa se pueden incluir servicios para rastrear y supervisar el estado dinámico de un dispositivo, administrar eventos y optimizar la operación de dispositivos autónomos. Los sistemas de acuaponía basados en loT permiten la visualización y el control remoto de los parámetros del sistema. Con el fin de garantizar el flujo oportuno de dichos parámetros, es indispensable garantizar un servicio de comunicación que permita recopilar en tiempo real los datos de los sensores y entregarlos, libres de errores, a los servicios de almacenamiento, visualización, análítica y big data. Los diversos parámetros se miden constantemente a través de nodos de sensor especificados. Los nodos son constantemente sondeados por una computadora de supervisión (Ojha, Misra y Raghuwanshi, 2015) (Del Rosario et al., 2011)

\subsection{Prototipo del servicio de inteligencia artificial}

Con el objetivo de crear un prototipo del servicio de inteligencia artificial, se tomaron los datos de Monsees, Kloas y Wuertz (2017), para un sistema acuapónico en aguas profundas (balsa flotante). Se tomaron los datos tanto del material vegetal, tomate (Solanum lycopersicum) como de la tilapia (Oreochromis mossambicus); adicionalmente se tomaron datos de la dinámica de algunos de los compuestos metabólicos $\left(\mathrm{NO}_{3}{ }^{-}, \mathrm{NO}_{2}^{-}\right.$y $\left.\mathrm{NH}_{4}{ }^{+}\right)$que influyen en el rendimiento del proceso.

\section{Datos capturados por loT}

Para el prototipo, se tomaron 1990 registros de datos con las condiciones de cría (oxígeno disuelto, $\mathrm{pH}$, temperatura y conductividad) en las unidades de peces (RAS) e hidropónicas (Hydro) de una referencia de acuicultura convencional (A), un sistema acuapónico acoplado (C) y un sistema acuapónico desacoplado (D), evaluado durante el período experimental de 154 días. En el cuadro 2 se presentan algunas visualizaciones de los datos analizados (Monsees, Kloas, Wuertz, 2017).

Cuadro 2

Visualizaciones de los datos analizados

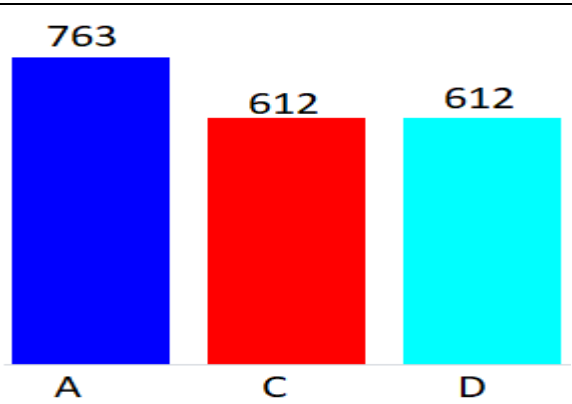

Sistemas acuicultura convencional $(A)$, sistemas acuapónicos acoplados (C) y sistemas acuapónicos desacoplados (D).

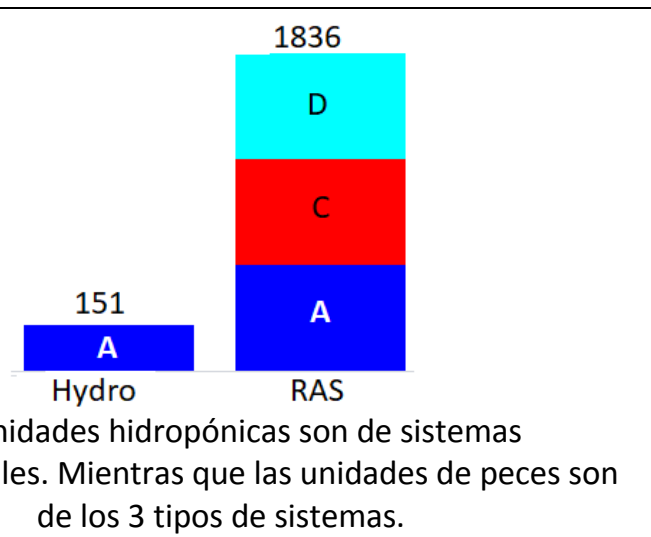
de los 3 tipos de sistemas. 


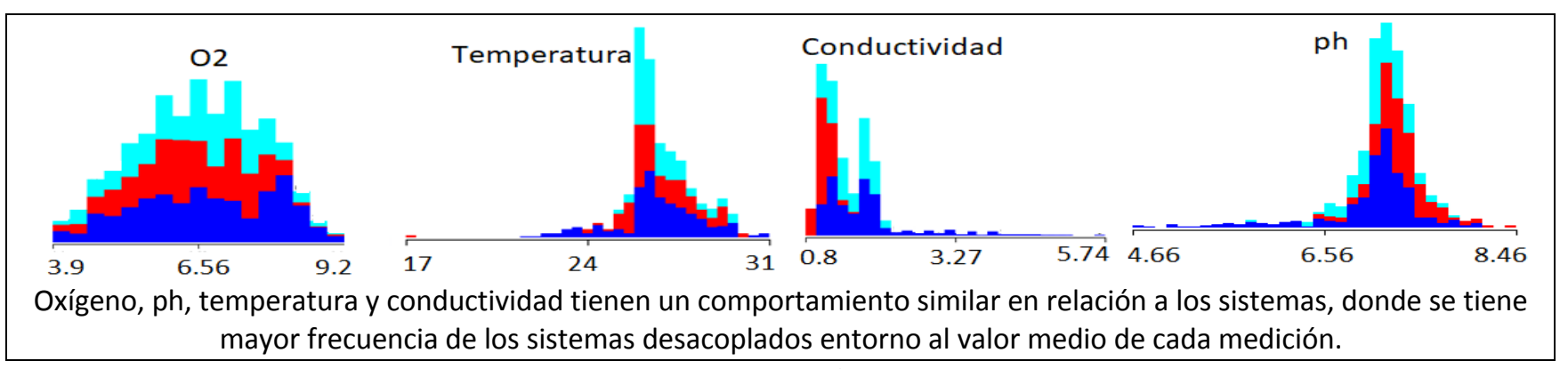

Fuente: Elaboración propia

\section{Aprendizaje no supervisado para identificar similitudes}

Con los datos se realizó un análisis con aprendizaje no supervisado de máquinas, específicamente un análisis de clustering para buscar similaridades entre las mediciones con el algoritmo de K-means. En el cuadro 3 se describen los 4 cluster encontrados.

El Cluster 1 está conformado por 612 datos de unidades de peces (RAS), sistemas convencionales (A), con un promedio de oxígeno en 6.29, $\mathrm{pH}$ en 7.30 , temperatura 26.75 y conductividad en 1.19.

El Cluster 2 está conformado por 612 datos de unidades de peces (RAS), sistemas desacoplados (D), con un promedio de oxígeno en 6.32 , pH en 7.20, temperatura 27.27 y conductividad en 1.53 .

El Cluster 3 está conformado por 151 datos de unidades hidropónicas (Hydro), sistemas desacoplados (A), con un promedio de oxígeno en 8.14 , pH en 6.38, temperatura 24.37 y conductividad en 3.11 .

Finalmente, el Cluster 4 está conformado por 612 datos de unidades de peces (RAS), sistemas acoplados (C), con un promedio de oxígeno en 6.53 , pH en 7.11, temperatura 26.83 y conductividad en 1.53 .

Cuadro 3

Resultado del análisis de clustering

\begin{tabular}{|c|c|c|c|c|}
\hline & $\begin{array}{c}\text { Cluster 1 } \\
\text { Tamaño: 612 }\end{array}$ & $\begin{array}{c}\text { Cluster 2 } \\
\text { Tamaño: 612 }\end{array}$ & $\begin{array}{c}\text { Cluster 3 } \\
\text { Tamaño: 151 }\end{array}$ & $\begin{array}{c}\text { Cluster 4 } \\
\text { Tamaño: 612 }\end{array}$ \\
\hline RAS/Hydro & RAS & RAS & Hydro & RAS \\
\hline System & A & D & D & C \\
\hline $\mathrm{O}_{2}$ & 6,29 & 6,32 & 8,14 & 6,53 \\
\hline $\mathrm{pH}$ & 7,30 & 7,20 & 6,38 & 7,11 \\
\hline Temperature & 26,75 & 27,27 & 24,37 & 26,83 \\
\hline Conductivity & 1,19 & 1,53 & 3,11 & 1,53 \\
\hline
\end{tabular}

Fuente: Elaboración propia

\section{Aprendizaje supervisado para predecir comportamiento del pH}

Dentro de las condiciones ambientales que se necesitan para la producción de Tilapia, el agua es vital tanto para el mantenimiento como para la productividad del pez; esta se evalúa por medio de las propiedades físicoquímicas, entre las más importantes destacan: temperatura, concentración de oxígeno, pH; en lo referente a la temperatura, esta no debe estar por debajo de $24{ }^{\circ} \mathrm{C}$, el pH entre 6.5 y 9; por su parte el oxigeno no debe tener una concentración menor a 4.5 mg/ L ( Saavedra Martínez, 2006, Sierra y Jenaro, 2019); de acuerdo a lo anterior, con un sistema predictivo el sistema podría ser controlado cuando los variables estén por fuera del rango óptimo de crecimiento o podrían predecirse, en caso de que se quisiera hacer una planeación de la producción en ciertos periodos del año. Se construye por medio de técnicas de aprendiazje supervisado de máquinas un modelo predictivo del $\mathrm{pH}$ que permita tomar medidas preventivas. Para realizar la predicción se discretizó el pH en 3 rangos: Bajo (4.6-5.9), medio (6.0 - 7.1) y alto (7.2-8.5). Para realizar la predicción del pH se utilizó el algoritmo 
de árboles de decisión, el cual crea una gráfica identificando los nodos más relevantes en la parte superior del árbol. Al descender por los nodos se llega a la predicción del pH en las hojas del árbol. En la gráfica 1 se presenta una muestra del ábol encontrado, logrando una exactitud del $80 \%$, es decir que se acierta el $80 \%$ de las predicciones. En la rama amarilla se encuentran unidades hidropónicas con temperatura menor a 25, oxígeno menor a 8.23, cuya predicción es pH en el rango (4.6-5.9). En la rama azul se tienen unidades para peces, en sistemas desacoplados, conductividad menor a 1.2, temperatura mayor a 26.3 , cuya predicción pH está en el rango(7.2-8.5).

\section{Gráfica 1}

Arbol de decisión para la predicción del pH

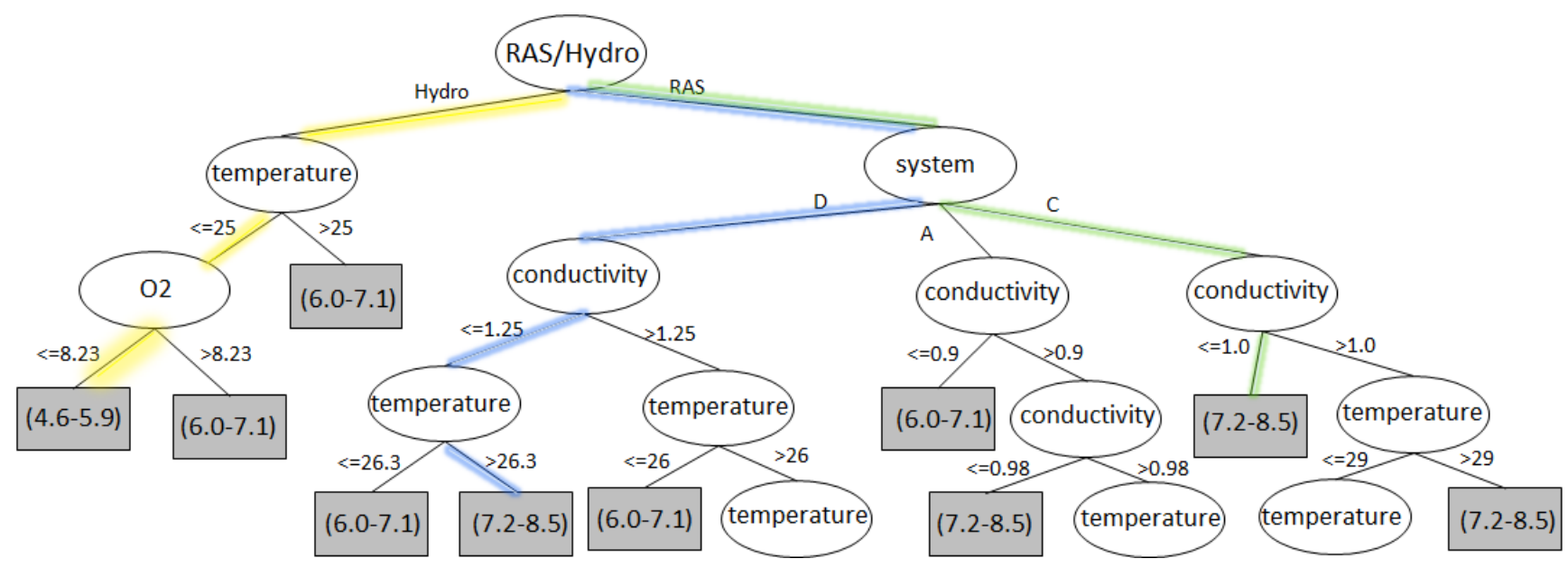

Fuente: Elaboración propia

Cuando la predicción de pH está en el rango más bajo (4.6-5.9) se recomienda subir el pH, adicionando una base o aumentando el tiempo de residencia del agua en las plantas. Cuando el rango del pH está en el rango medio (6.0-7.1) el sistema funciona bien y la idea es que se mantenga el máximo tiempo postible Finalmente, cuando la predicción del pH está en el rango alto (7.2-8.5) se recomienda adcionar un acido o aumentar el flujo de agua desde las plantas al sistema de peces.

\section{Conclusiones}

La acuaponía se presenta como una alternativa para la producción de diversos grupos de alimentos, destacando el pescado que es una de las mejores fuentes de proteína; este tipo de sistema de producción de alimentos se puede hacer tanto en el sector rural como el urbano, es de fácil implementación y además hace un uso sostenible del agua de acuerdo a los requerimientos de la comunidad impactada.

Para aumentar su productividad y eficiencia, se requiere de un seguimiento continuo, el cual se puede llevar a cabo con un dispositivo móvil, gracias a los sensores y al uso de arquitecturas de loT ya utilizados en sistemas de agricultura de precisión, los cuales se pueden instalar fácilmente y a un costo razonable, permitiendo además controlar la calidad y economizar insumos.

Para el monitoreo de variables relacionadas con el aire, suelo, agua, plantas y animales en un sistema de acuaponía, se propuso una arquitectura monitoreada con IOT e IA basada en 4 capas de sensado, comunicaciones, servicios y aplicaciones. Para el servicio de inteligencia artificial fue construido un prototipo con dos análisis: 1) aprendizaje no supervisado de máquinas para encontrar registros de datos similares y 2) aprendizaje supervisado de máquinas para predecir el comportamiento del $\mathrm{pH}$. Con el prototipo construido se encontró que al realizar las predicciones sobre valor del $\mathrm{pH}$, se pueden tomar acciones en el presente para 
controlar el sistema. Como trabajo futuro, se espera que el diseño propuesto pueda ser implementado a diferentes escalas.

\section{Referencias bibliográficas}

Addy, M. M., Kabir, F., Zhang, R., Lu, Q., Deng, X., Current, D., Griffith, R., Ma, Y., Zhou, W., Chen, P., \& Ruan, R. (2017). Co-cultivation of microalgae in aquaponic systems. Bioresource Technology, 245(August), 27-34. https://doi.org/10.1016/j.biortech.2017.08.151

Alberto, C., \& Nieto, S. (2019). Estrategias de manejo para mejorar la producción piscícola en un sistema de jaulas flotantes con Tilapia roja (oreochromis sp ), en Montería , Córdoba.

Aqeel-Ur-Rehman, A.; Islam, N. \& Shaikh, Z. A. (2014). A review of wireless sensors and networks' applications in agriculture. Computer Standards and Interfaces, 36(2), 263-270. Recuperado de: https://doi.org/10.1016/j.csi.2011.03.004

Atzori, L., A. lera; \& Morabito, G. (2010). The Internet of Things: A survey. Computer Networks54(15): p. 27872805.

Baclic, O., Tunis, M.; Young, K.; Doan, C.; Swerdfeger, H.; Schonfeld, J. ... \& Hub, I. (2020). Natural language processing (NLP) a subfield of artificial intelligence. CCDR, 46(6).

Badia-Melis, R., Mishra, P., \& Ruiz-García, L. (2015). Food traceability: New trends and recent advances. A review. Food Control, 57, 393-401. https://doi.org/10.1016/j.foodcont.2015.05.005

Bertino, E., \& Islam, N. (2017). Botnets and Internet of Things Security. Computer. https://doi.org/10.1109/MC.2017.62

Buzby, K. M., Waterland, N. L., Semmens, K. J., \& Lin, L. S. (2016). Evaluating aquaponic crops in a freshwater flow-through fish culture system. Aquaculture, 460, 15-24. https://doi.org/10.1016/j.aquaculture.2016.03.046

Caló, P. (2011). Introducción a la Acuaponia. Centro Nacional de Desarrollo Acuícola- CENADAC. Argentina. 15p

Channe, H., Kothari, S., \& Kadam, D. (2015). Multidisciplinary Model for Smart Agriculture using Internet - of Things ( IoT ), Sensors, Cloud - Computing, Mobile - Computing \& Big - Data Analysis. Int.J.Computer Technology \& Applications, 6(3), 374-382. https://pdfs.semanticscholar.org/e914/d431520f4cb60b9d80b123f7d43782c1aba8.pdf

Cisco Visual Networking Index (2017) Tomado de https://www.cisco.com/c/en/us/solutions/serviceprovider/vni-network-traffic-forecast/infographic.html

Consulting Hambrey. (2013). The relevance of aquaponics to the New Zealand aid programme, particularly in the Pacific (M. of F. A. and Trade (ed.); New Zealan, Issue December).

Contreras J y Mayol R.(2016) "Variación de parámetros de criptografía con curvas elípticas usados en la firma digital de datos sobre una red de sensores inalámbricos," Revista Ingeniería al Día, Universidad del Sinú, pp. 41-53, 2016.

Cor, N. V., Sjaak, W. \& Bedir, T. (2016). Internet of Things in Agriculture. Disponible en: https://iotiran.com/media/k2/attachments/Verdouwetal.reviewpaperloTinAgricultureCABReviewsdraft.p df 
Del Rosario, J.M., et al.(2011) Deployment of a wireless sensor network for aquaculture and lake resource management. In: IEEE 7th International Conference on Wireless and Mobile Computing, Networking and Communications (WiMob), Shanghai, P.R. China, pp. 433-438

Fennema, O. (2010). Quimica de los Alimentos. In S. Damodaran, K. Parkin, \& O. Fennema (Eds.), University of Wisconsin (3o). Acribia S.A.

Forchino, A. A., Lourguioui, H., Brigolin, D., \& Pastres, R. (2017). Aquaponics and sustainability: The comparison of two different aquaponic techniques using the Life Cycle Assessment (LCA). Aquacultural Engineering, 77, 80-88. http://doi.org/10.1016/j.aquaeng.2017.03.002

Gao, Z., Wanyama, T., Singh, I., Gadhrri, A., \& Schmidt, R. (2020). From Industry 4.0 to Robotics 4.0-A Conceptual Framework for Collaborative and Intelligent Robotic Systems. Procedia Manufacturing, 46, 591-599.

Garcia-Morchon, et al. (2017) State of the Art and Challenges for the Internet of Things Security, Internet Engineering Task Force (IETF).

Goddek, S., \& Keesman, K. J. (2018). The necessity of desalination technology for designing and sizing multiloop aquaponics systems. Desalination, 428(May 2017), 76-85.

https://doi.org/10.1016/j.desal.2017.11.024

Guerrero-Ibañez, J. A., Estrada-Gonzalez, F. P., Medina-Tejeda, M. A., Rivera-Gutierrez, M. G., Alcaraz-Aguirre, J. M., Maldonado-Mendoza, C. A., Toledozuñiga, D., \& Lopez-Gonzalez, V. I. (2017). SGreenH-loT: Plataforma loT para Agricultura de Precisión. Sistemas Cibernetica e Informatica, 14, 53-58.

Gutierrez Leon, E., Montiel Arguijo, J. E., Carreto Arellano, C., \& Menchaca García, F. R. (2019). Propuesta de sistema de gestión inteligente basado en loT para hidroponia. Research in Computing Science, 148(10), 219-233. https://doi.org/10.13053/rcs-148-10-19

Kubo. (2015)The research of loT based on RFID technology. En 7th International Conference on Intelligent Computation Technology and Automation, ICICTA 2014. 2015. Institute of Electrical and Electronics Engineers Inc.

Li, S., Tryfonas, T., \& Li, H. (2016). The Internet of Things: a security point of view. Internet Research, 26(2), 337-359. https://doi.org/10.1108/IntR-07-2014-0173

Microsoft. (2015). FarmBeats: Al, Edge \& loT for Agriculture - Microsoft Research. https://www.microsoft.com/en-us/research/project/farmbeats-iot-agriculture/

Minsalud. (2015). Encuesta Nacional de la Situación Nutricional - ENSIN 2015. Retrieved from https://www.minsalud.gov.co/sites/rid/Lists/BibliotecaDigital/RIDE/VS/ED/GCFI/presentacion-ensin-2017medellin.pdf

Monsees, H., Kloas, W. \& Wuertz, S. (2017). Decoupled systems on trial: Eliminating bottlenecks to improve aquaponic processes (Open Access). LoS ONE, 12 (9), art. no. e0183056. Recuperado de: http://journals.plos.org/plosone/article/file?id=10.1371/journal.pone.0183056\&type=printable doi: 10.1371/journal.pone.0183056

Montoya, E. A. Q., Colorado, S. F. J., Muñoz, W. Y. C., \& Golondrino, G. E. C. (2017). Propuesta de una Arquitectura para Agricultura de Precisión Soportada en IoT. RISTI - Revista Iberica de Sistemas e Tecnologias de Informacao, (24), 39-56. https://doi.org/10.17013/risti.24.39-56 
Nelson, R. L., and J. S. Pade.( 2006). Nutrient film technique aquaponics. Aquaponics Journal. No. 42. pp: 18-21. Nelson, L. R. 2008. Aquaponics Food Production. Raising Fish and Profit.

Odema, M., Adly, I., Wahba, A. \& Ragai, H. (2018) Smart Aquaponics System for Industrial Internet of Things (IloT). In: Advances in Intelligent Systems and Computing, vol 639. Springer, Cham

Ojha, T., Misra, S., \& Raghuwanshi, N. S. (2015a). Wireless sensor networks for agriculture: The state-of-the-art in practice and future challenges. Computers and Electronics in Agriculture, 118, 66-84. https://doi.org/10.1016/j.compag.2015.08.011

Ojha, T., Misra, S., \& Raghuwanshi, N. S. (2015b). Wireless sensor networks for agriculture: The state-of-the-art in practice and future challenges. Computers and Electronics in Agriculture, 118, 66-84. https://doi.org/10.1016/j.compag.2015.08.011

Ojha, T., S. Misra, \& N.S. Raghuwanshi. (2015). Wireless sensor networks for agriculture: The state-

Pérez, L. (2007). Hidroponía en Casa. (Ed). Corazón Verde; San José, Costa Rica. 54 p

Pérez-Freire, L., et al.(2015). Smart Farming and Food Safety Internet of Things Applications - Challenges for Large Scale Implementations. 2015, AIOTI WG06. p. 49.

Primo Yufera, E. (1998) Química de los Alimentos. Editorial Síntesis.

Project FEED 1010. (2016). Components of an Aquaponics System. Institute for System Biology. http://www.projectfeed1010.com/blog/2014/07/15/aquaponics-components/

Quiroga, E., Jaramillo, S., Campo, W., \& Ganchi, G. (2017). Propuesta de una Arquitectura para Agricultura de Precisión Soportada en loT. RISTI - Revista Iberica de Sistemas e Tecnologias de Informacao, 24, 39-56. https://doi.org/10.17013/risti.24.39-56

Ran, N. (2014). Design and implementation of intelligent greenhouses based on the internet of things. En Applied Mechanics and Materials. 2014. p. 188-191.

Ruiz-Garcia, L., et al. (2009) A Review of Wireless Sensor Technologies and Applications in Agriculture and Food Industry: State of the Art and Current Trends. Sensors, 2009. 9(6).

S. Al Salami, J. Baek, K. Salah, \& E. Damiani. (2016) "Lightweight encryption for smart home," in Proceedings 2016 11th International Conference on Availability, Reliability and Security, ARES 2016, 2016, pp. 382-388.

S. Al Salami, J. Baek, K. Salah, \& E. Damiani. (2016) “Lightweight encryption for smart home," in

S. Nguyen \& C. Rong. (2007). "ZigBee Security Using Identity-Based Cryptography", Autonimic and Trusted Computing, vol. 4610, pp. 3-12, 2007.

Saavedra Martínez, M. (20 C.E.). Manejo del cultivo de tilapia. Managua, Nicaragua. In Manual.

Scalera, A., et al. (2013).From animal monitoring to early warning systems through the Internet of Things. En Precision Livestock Farming 2013 - Papers Presented at the 6th European Conference on Precision Livestock Farming, ECPLF 2013.

Somervillle, C., Cohen, M., Pantaella, E., Stankus, A., \& Lovatelli, A. (2014). Small-scale aquaponic

Sundmaeker, H., et al.(2020). Internet of Food and Farm 2020. En Digitising the Industry O. Vermesan, Friess, Peter, Editor. 2016, River Publishers. p. 129-150. 
Talavera, J. M., Tobón, L. E., Gómez, J. A., Culman, M. A., Aranda, J. M., Parra, D. T., ... Garreta, L. E. (2017). Review of loT applications in agro-industrial and environmental fields. Computers and Electronics in Agriculture, 142(118), 283-297. https://doi.org/10.1016/j.compag.2017.09.015

Talavera, J. M., Tobón, L. E., Gómez, J. A., Culman, M. A., Aranda, J. M., Parra, D. T., ... Garreta, L. E. (2017). Review of loT applications in agro-industrial and environmental fields. Computers and Electronics in Agriculture, 142(118), 283-297. https://doi.org/10.1016/j.compag.2017.09.015

Tao, R., et al. (2014). Secure gateway of internet of things based on appweb and secure sockets layer for intelligent granary management system. En IFIP Advances in Information and Communication Technology. 2014. p. $78-89$

Tzounis, A., Katsoulas, N., Bartzanas, T., \& Kittas, C. (2017). Internet of Things in agriculture, recent advances and future challenges. Biosystems Engineering, 164, 31-48.

https://doi.org/10.1016/j.biosystemseng.2017.09.007

Valencia, A. G., David, J., \& Soto, R. (2016). Piscícolas Cultivo de Trucha y Tilapia. http://www.corantioquia.gov.co/SiteAssets/PDF/Gestión ambiental/Producción y Consumo Sostenible/Manuales_GIRH/Pisicola.pdf

Verdouw, C.N., et al. (2016). Virtualization of food supply chains with the internet of things. Journal of Food Engineering, 2016. 176: p. 128-136.

Villadomat, L. (2012). Handbook for construction and operation of domestic scale aquaponic systems in the West Bank. (R. Capitale, Ed.) (Asal). Roma: Oxfarm.

Yigit, E., Sabanci, K., Toktas, A., \& Kayabasi, A. (2019). A study on visual features of leaves in plant identification using artificial intelligence techniques. Computers and electronics in agriculture, 156, 369-377.

Yu, F. R., \& He, Y. (2019). Introduction to Machine Learning. In Deep Reinforcement Learning for Wireless Networks (pp. 1-13). Springer, Cham.

Zhao, G., et al. (2015) Applied research of IOT and RFID technology in agricultural product traceability system, En IFIP Advances in Information and Communication Technology, D. Li and Y. Chen, Editors. 2015, Springer New York LLC. p. 506-514.

Esta obra está bajo una Licencia Creative Commons Attribución-NoCommercial 4.0 International

\section{(cc) BY-NC}

